\title{
Root Coverage with a New Collagen Matrix and Coronally Advanced Flap: A Case Report
}

\section{Paulo Sergio Henriques*}

Department of Periodontics, Sao Leopoldo Mandic School of Dentistry, Campinas, SP, Brazil

\begin{abstract}
Background: Gingival recession is a frequent finding in patients. The connective tissue graft plus coronally advanced flap $(\mathrm{CTG}+\mathrm{CAF})$ is considered the gold standard for root coverage therapy. An alternative option, both avoiding the need for palatal donor tissue and allograft material, is the use of collagen matrix (CM) of porcine origin.

Methods: The aim of this report is to describe a surgical utilizing collagen matrix plus coronally advanced flap $(C M+C A F)$ in a $3 \mathrm{~mm}$ buccal gingival recession associated to traumatic brushing in the maxillary left canine. The goal of treatment was determines if a CM with CAF might be as effective in the root coverage procedure of Miller's class I recession defect.

Results: Gradual surgical healing with minimal postoperative morbidity and very slight discomfort was observed at 1 week. The clinical observation at 12-months revealed complete root coverage with an adequate zone of keratinized tissue with good healing, color, and texture nicely at the native adjacent soft tissues.

Conclusions: Patient satisfaction and esthetics was very high. The results seem to suggest that $\mathrm{CM}+\mathrm{CAF}$ can provide a valid treatment procedure in root coverage. Moreover, it has shown a significant reduction in surgery time, maintenance of marginal tissue health, and mainly patient morbidity without the graft harvest.
\end{abstract}

Keywords: Gingival recession; Root coverage; Collagen matrix; Tissue regeneration

Abbreviations: CTG+CAF: Connective Tissue Graft Plus Coronally Advanced Flap; CM: Collagen Matrix; CEJ: Cemento-Enamel Junction

\section{Introduction}

The treatment of gingival recession is a common requirement due to aesthetic concern or root sensitivity in patients with high standards of oral hygiene [1]. Gingival recession is defined as an apical shift of the gingival margin from its position $1 \mathrm{~mm}$ coronal to or at the level of the cemento-enamel junction (CEJ) with exposure of the root surface to the oral environment [2].

Tooth brushing trauma is the primary etiologic factor for gingival recession; in this situation cervical abrasion defects are frequently associated with the root exposures [3]. In the last decades, different surgical procedures were proposed to obtain root coverage: Pedicles flaps (PF), connective tissue graft (CTG), guided tissue regeneration, coronally advanced flaps (CAF), CAF+CTG, and more recently $\mathrm{CAF}+\mathrm{CTG}$ plus enamel matrix derivative [4-9]. The coronally advanced flap (CAF) is a very common approach for root coverage, the surgery does not involve a palatal donor site, and it was demonstrated to be a safe and predictable. Localized gingival recessions have been successfully treated with this technique [10]. Outcomes have been reported by adding a connective tissue graft to the coronally advanced flap $(\mathrm{CAF}+\mathrm{CTG})$

Due the morbidity and time associated with soft tissue graft harvest and the limited supply, the acellular dermal matrix is an important substitute. However, because this allograft material is derived from human cadavers, it is associated with ethical concerns, possible risk of disease transmission, extensive shrinkage during the healing period and is not completely incorporated histologically.

An alternative option, both avoiding the need for palatal donor tissue and allograft material, is the use of collagen matrix (CM) of porcine origin (Mucograft ${ }^{\circledR}$, Geistlich, Wolhusen, Switzerland). Recently, a new two-layer, xenogeneic $\mathrm{CM}$ has been cleared by the
US Food and Drug Administration for regenerative therapy involving teeth and implants, including treatment of dehiscence defects around teeth. Fabricated as a matrix and composed of pure porcine collagen obtained by standardized, controlled manufacturing processes. The collagen was extracted from veterinary-certified pigs and purified to avoid antigenic reactions. The matrix was made of collagen type I and type III without further cross-linking or chemical treatment and sterilized in double blisters by gamma irradiation. CM has two layers and is approximately $2.5 \mathrm{~mm}$ thick. The first layer is a compact layer, facing the oral cavity, consisting of a denser collagen that protects the wound but allows tissue adherence for favorable wound healing. This layer has a smooth texture with appropriate properties to accommodate suturing to the host mucosal margins. The second layer is a thicker, porous collagen that encourages tissue integration. This porous surface is placed adjacent to the host tissue to facilitate organization of the blood clot and promote neoangiogenesis [11,12]. Because CM seems to be a promising soft tissue graft substitute, we decided to test in this case report whether its placement under a CAF in a recession defects support root coverage.

\section{Case Report}

In January 2013, a 36-year-old women, presented at the Periodontology Clinic at São Leopoldo Mandic School of Dentistry for consultation regarding root hyper sensibility and in satisfaction

*Corresponding author: Paulo Sergio Henriques, Head of Department, Department of Periodontics, Sao Leopoldo Mandic School of Dentistry, Rua. Dr. Jose B. C. Nogueira 214, Sala 125, VI. Madalena, Campinas, S. Paulo, 13091-611, Brazil, Tel: +55-19-32550288; Fax: 0055 32944815; E-mail: phenriques@mpc.com.br

Received April 17, 2014; Accepted March 18, 2015; Published March 23, 2015

Citation: Henriques PS (2015) Root Coverage with a New Collagen Matrix and Coronally Advanced Flap: A Case Report. Dentistry 5: 292. doi:10.4172/21611122.1000292

Copyright: $\odot 2015$ Henriques PS. This is an open-access article distributed under the terms of the Creative Commons Attribution License, which permits unrestricted use, distribution, and reproduction in any medium, provided the original author and source are credited. 
esthetic in a $3 \mathrm{~mm}$ buccal gingival recession associated to traumatic brushing in the maxillary left canine (Figure 1a). Her medical history was unremarkable, no contraindications for periodontal surgery and no talking medications known to interfere with periodontal tissue healing, and she denied any history of smoking and collagen allergic. Periapical radiograph was taken, in a standardized manner using the long-cone paralleling technique.

The patient agreed to participate in this study and gave their written informed consent on an Institutional Review Board consent form. The study protocol involved a initial therapy to establish optimal plaque control and gingival health conditions, surgical therapy, a maintenance phase, and a postoperative evaluation 2, 4, 6 and 12 months after the surgery. Clinical photographs were taken at baseline, at surgery and each follow-up visit.

A periodontal examination was performed and all clinical measurements were determined to the nearest millimeter using a UNC15 periodontal probe (Hu-Friedy, Chicago, IL, USA). Vertical probing measures were made at the mid-buccal aspect of canine measured from the CEJ to the free gingival margin. All measures parameters were recorded at baseline included gingival recession depht $(3 \mathrm{~mm})$, probing depth $(2 \mathrm{~mm})$ and clinical attachment level $(5 \mathrm{~mm})$. The width of keratinized tissue $(3 \mathrm{~mm}$ ) was determined from gingival margin to the muco-gingival junction (MGJ) that was accomplished by coronal displacement of the alveolar mucosa against a horizontally positioned periodontal probe.

The goal of treatment was determines if a CM with CAF might be as effective in the root coverage procedure of Miller's class I recession defect. The patient was offered the option the coronally advanced flap plus connective tissue graft $(\mathrm{CAF}+\mathrm{CTG})$ is considered the gold standart procedure. Because the morbidity and time associated with soft tissue graft harvest, $\mathrm{CM}+\mathrm{CAF}$ were chosen.

\section{Surgical Procedure}

Preoperative intra-oral antisepsis was accomplished using $0.12 \%$ chlorhexidinedigluconate solution rinsed for $1 \mathrm{~min}$. Following administration of local anesthesia with $2 \%$ mepivacaine containing
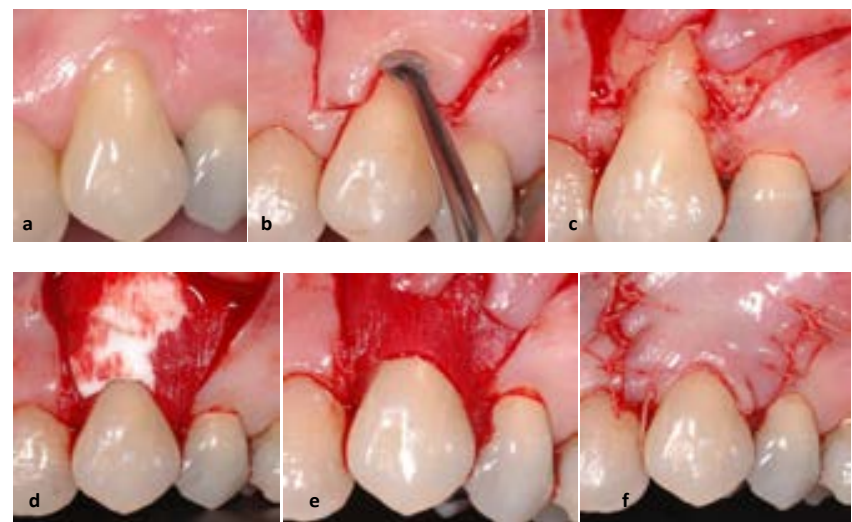

Figure 1: (a) Preoperative view of the Miller Class I gingival recession on the buccal of the maxillary left canine. (b) A CAF was performed and the soft tissue apical to the root exposure elevated (3.0 to $4.0 \mathrm{~mm}$ ) full thickness by inserting a periosteum elevator. (c) The vertical incisions were elevated split thickness and the anatomic interdental papillae were deepithelialized to create connective tissue beds. (d) CM was trimmed to extend 2.0 to $3.0 \mathrm{~mm}$ beyond the bone crest (both laterally and apically). (e)Collagen matrix fixed with a sling suture over the recession defect. (f) Coronally advanced flap, completely covering the matrix. epinephrine 1:100,000, the root surface was gently scaled and planed with Gracey curettes(Hu-Friedy, Chicago, IL, USA), which contributed to reduce buccal prominence, and conditioned with 24\% EDTA gel for $2 \mathrm{~min}$ to remove the smear layer. The exposed root surface was rinsed abundantly with sterile saline solution to remove all EDTA residues.

The surgical technique used to achieve soft tissue coverage was CAF. Two oblique, divergent beveled incisions were performed at the mesial and distal line angles of the two peripherical teeth with gingival recession. These incisions, together with the intrasulcular incisions along the mesial and distal recession margins, designed the two external surgical papillae. Crossed submarginal incisions, made interproximally, created the interdental surgical papillae.

The soft tissue apical to the root exposure (including the residual keratinized tissue) was elevated full thickness by inserting a small periosteum elevator into the probeable sulcus and proceeding in the apical direction to expose 3.0 to $4.0 \mathrm{~mm}$ of bone apical to the bone dehiscence. This was done to include the periosteum and the maximum soft tissue thickness in the central portion of the flap covering the avascular root exposure (Figure 1b).

The vertical incisions were elevated split thickness, keeping the blade almost parallel to the bone plane, thus leaving the periosteum to protect the underlying bone in the lateral areas of the flap. Apical to the bone exposure, split-thickness flap elevation continued until it was possible to move the flap passively in the coronal direction. To permit the coronal advancement of the flap, all muscle insertions present in the thickness of the flap were eliminated. This was done keeping the blade parallel to the external mucosal surface. Coronal mobilization of the flap was considered adequate when the marginal portion of the flap was able to passively reach a level coronal to the CEJ of the recession defects. The flap should be stable in its final coronal position, even without the sutures. Once coronally advanced, the flap partially overlaid the soft tissues mesial and distal to the receiving bed. These areas and the facial soft tissue of the anatomic interdental papillae were deepithelialized to create connective tissue beds (Figure 1c).

$\mathrm{CM}$ test material was trimmed to extend 2.0 to $3.0 \mathrm{~mm}$ beyond the bone crest (both laterally and apically) (Figure 1d) and fixed with a sling suture using a 5-0 sutures (Vicryl, Johnson \& Johnson, S. J. Campos, Brazil) around the crown of the tooth (Figure 1e). The flap was coronally positioned $2.0 \mathrm{~mm}$ above the CEJ to fully cover the $\mathrm{CM}$ by suturing it to the de-epithelialized papilla regions. At all times caution was maintained to avoid over compression of the test material. Suturing of the flap started with two interrupted periosteal 5-0 sutures at the most apical extension of the vertical incisions; it proceeded coronally with other interrupted sutures, each of them directed from the flap to the adjacent buccal soft tissue, in the apical-coronal direction. This was done to facilitate the coronal displacement of the flap and to reduce the tension of the flap. The sling sutures permitted stabilization of the surgical papillae over the interdental connective tissue beds and allowed for a precise adaptation of the flap margin over the convexity of the underlying anatomic crowns. At the end of the surgery, the flap margin was coronal to the CEJ (Figure 1f). This was done to compensate for post-surgical soft tissue shrinkage.

No periodontal dressing was applied. No antibiotic was prescribed. Acetaminophen $750-\mathrm{mg}$ as needed for pain was noted. The patient was instructed to rinse three times a day for 1 minute with $0.12 \%$ chlorhexidine digluconate solution during 4 weeks. Rapid surgical healing with minimal postoperative morbidity was observed at 1 week (Figure 2a). The sutures were removed 14 days after surgery. 

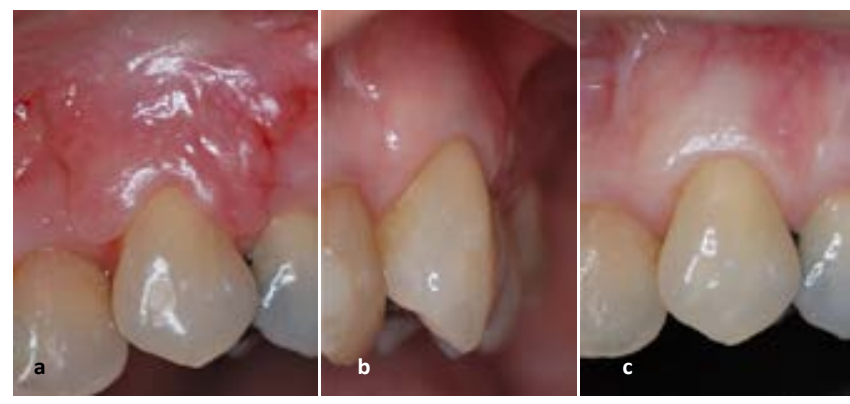

Figure 2: (a) Good healing at 7 days postoperative. (b) At 12 months following surgery, lateral view showing root coverage. (c) Root coverage and healthy soft tissues are evident at twelve months post-surgery.

Subject was advised to practice a care mechanical oral hygiene 4 weeks following surgery to minimize trauma to the surgical site and after this period, instructed in the Bass technique with an ultrasoft toothbrush and monitored once every 2 months until the end of the study at 12 months. During this period received professional supra-gingival plaque control. The patient reported very slight discomfort.

The clinical observation at 12 -months revealed complete root coverage with an adequate zone of keratinized tissue with good healing, tissue contour without adverse sequelae (like keloids), color, and texture, nicely at the native adjacent soft tissues (Figures $2 \mathrm{~b}$ and $2 \mathrm{c}$ ).

\section{Discussion}

This report documents the use of $\mathrm{CM}+\mathrm{CAF}$ for root coverage. This surgical procedure was designed to be shorter, less aggressive, and was thought to have fewer postoperative complications. Recently published prospective clinical trials investigating the efficacy of CM in treating both keratinized mucosal deficiencies and gingival recession defects suggesting that $\mathrm{CM}$ may provide, viable alternative to autogenous tissue grafts and unlimited "off-the-shelf" supply of grafting material, reducing surgery time by approximately one-third. The savings in time and discomfort is weighed against the cost of the matrix $[11,12]$.

Despite esthetics being considered the primary goal of rootcoverage procedures, few studies evaluated the changes of esthetic conditions as they related to the opinions of patients. In these studies, the patient was satisfied with the final esthetic result. The incidence of adverse effects, such as discomfort with or without pain, was directly related to donor sites of CTG [12]. Also, procedures that made a reduction in the operatory time, eliminated the need for a second surgical site and its associated morbidity and used smaller palatal grafts was better accepted [13].

An in vitro testing of this collagen matrix showed the in-growth of primary human fibroblasts into the $\mathrm{CM}$, which resulted in an increased expression of extracellular matrix proteins such as collagen type I and fibronectin. Recently, CM and another prototype with a different source of collagen were compared in a non-submerged healing environment in combination with the apically repositioned flap. Clinical results demonstrated an increase in the width and thickness of the KT. The qualitative histological analysis revealed complete healing of both CM, resulting in mature mucosal and submucosal tissues.

In an experimental study, the combination of a $\mathrm{CM}$ and the $\mathrm{CAF}$ procedure significantly reduced the recession and increased the width of KT. Histologically, allowed an uneventful healing, the matrix being completely incorporated into the adjacent host connective tissues, in the absence of a significant inflammatory response. The healing was characterized by the formation of new cementum and new connective tissue attachment in the apical aspect of the defect and by a junctional epithelium in its most coronal third. When compared with the CAF alone, the CM graft attained more tissue regeneration, with a shorter epithelium and a larger new cementum formation [14].

An in vivo evaluation of Mucograft ${ }^{\bullet}$ minimal inflammation and no multinucleated giant cells was present. The material persisted in the tissue throughout the study. At the same research, results demonstrate great potential to reverse tissue recession and promote more healthy gingival tissue [15]. Collagen matrix can enhance oral soft tissue healing compared with spontaneous healing during the first week, based on clinical observations that the two distinct structures paved the way to improve the healing by an early stabilization of the coagulum (matrix function) [16].

\section{Summary}

This present study seems to suggest that $\mathrm{CM}+\mathrm{CAF}$ can provide a valid treatment procedure in Miller's class I root coverage and CM may offer a new option to CAF alone and palatal harvest. The need for twostage surgery was eliminated with a significant reduction in surgery time, no pain related and less morbidity presented. More extensive, long term clinical studies are needed to support this result obtained.

\section{Acknowledgments}

The author would like to thank the Geistlich Pharma Brazil, that supported in part this study granting a collagen matrix and gratefully acknowledge the help of $\mathrm{Dr}$. Marcelo Nunes for his assistance with the surgeries procedures. The author report no conflicts of interest related to this study.

\section{References}

1. American Academy of Periodontology (1996) Consensus report on mucogingival therapy. Proceedings of the World Workshop in Periodontics. Ann Periodontol 1: 702-706.

2. Wennstrom JL (1994) Proceedings of the $1^{\text {st }}$ European Workshop on Periodontology Mucogingival surgery In: Lang NP, Karring T, eds. Quintessence Publishing, Berlin; 193-209.

3. Zucchelli G, Mele M, Stefanini M, Mazzotti C, Mounssif I, et al. (2010) Predetermination of root coverage. J Periodontol 81: 1019-1026.

4. Harris RJ, Harris AW (1994) The coronally positioned pedicle graft with inlaid margins: a predictable method of obtaining root coverage of shallow defects. In J Periodontics Restorative Dent 14: 228-241.

5. Langer B, Langer L (1985) Subepithelial connective tissue graft technique for root coverage. J Periodontol 56: 715-720.

6. Prato GP, Clauser C, Magnani C, Cortellini P (1995) Resorbable membrane in the treatment of human buccal recession: a nine-case report. Int J Periodontics Restorative Dent 15: 258-267.

7. de Sanctis M, Zucchelli G (2007) Coronally advanced flap: a modified surgical approach for isolated recession-type defects: three-year results. $\mathrm{J}$ Clin Periodontol 34: 262-268.

8. Cortellini P, Tonetti M, Baldi C, Francetti L, Rasperini G, et al (2009) Does placement of a connective tissue graft improve the outcomes of coronally advanced flap for coverage of single gingival recessions in upper anterior teeth? A multi-centre, randomized, double blind, clinical trial. J ClinPeriodontol 36: $68-79$

9. Pilloni A, Paolantonio M, Camargo PM (2006) Root coverage with a coronally positioned flap used in combination with enamel matrix derivative: 18-month clinical evaluation. J Periodontol 77: 2031-2039.

10. Nieri M, Rotundo R, Franceschi D, Cairo F, Cortellini $P$, et al. (2009) Factors affecting the outcome of the coronally advanced flap procedure: a Bayesian network analysis. J Periodontol 80: 405-410.

11. Sanz M, Lorenzo R, Aranda JJ, Martin C, Orsini M (2009) Clinical evaluation of a new collagen matrix (Mucograft $₫$ prototype) to enhance the width of 
Citation: Henriques PS (2015) Root Coverage with a New Collagen Matrix and Coronally Advanced Flap: A Case Report. Dentistry 5: 292. doi:10.4172/2161-1122.1000292

keratinized tissue in patients with fixed prosthetic restorations: a randomized prospective clinical trial. J ClinPeriodontol 36: 868-876.

12. McGuire MK, Scheyer ET (2010) Xenogeneic collagen matrix with coronally advanced flap compared to connective tissue with coronally advanced flap for the treatment of dehiscence-type recession defects. J Periodontol 81: 11081117.

13. Cairo F, Nieri M, Cattabriga M, Cortellini P, De Paoli S, et al. (2010) Root coverage esthetic score after treatment of gingival recession: an interrater agreement multicenter study. J Periodontol 81: 1752-1758.

14. Vignoletti F, Nuñez J, Discepoli N, De Sanctis F, Caffesse R, et al. (2011)
Clinical and histological healing of a new collagen matrix in combination with the coronally advanced flap for the treatment of Miller class-I recession defects: an experimental study in the minipig. J Clin Periodontol 38: 847-855.

15. Ghanaati S, Schlee M, Webber MJ, Willershaugen I, Barbeck M, et al. (2011) Evaluation of the tissue reaction to a new bilayered collagen matrix in vivo and its translation to the clinic. Biomed. Mater 6: 015010.

16. Thoma DS, Sancho-Puchades M, Ettlin DA, Hammerle CH, Jung RE (2012) Impact of a collagen matrix on early healing, aesthetics and patient morbidity in oral mucosal wounds - a randomized study in humans. J Clin Periodonto 39: 157-165. 